This is the final peer-reviewed accepted manuscript of:

Wu, Y., and Carricato, M. 2018. Line-symmetric motion generators. Mechanism and Machine Theory, 127, pp. 112-125.

ISSN 0094-114X

The final published version is available online at:

https://doi.org/10.1016/i.mechmachtheory.2018.05.007

(C) 2018. This manuscript version is made available under the Creative Commons AttributionNonCommercial-NoDerivs (CC BY-NC-ND) 4.0 International License

(http://creativecommons.org/licenses/by-nc-nd/4.0/) 


\title{
Line-Symmetric Motion Generators ${ }^{\text {th }}$
}

\author{
Yuanqing $\mathrm{Wu}^{*}$ and Marco Carricato \\ University of Bologna, Bologna 40136, Italy
}

\begin{abstract}
When a rigid body is axially reflected through a moving line, its image undergoes a so-called line-symmetric motion. The space comprising all possible line-symmetric motions that share a common initial line is a four-dimensional submanifold, denoted $\mathrm{M}_{4}$, in the special Euclidean group $\mathrm{SE}(3)$. Recently, we showed that $\mathrm{M}_{4}$ may be used to characterize motions of a line-symmetric body that are free of self-spin and sliding, thus lending itself to applications such as remote center of motion devices for minimal invasive surgery and haptic interfaces. Aiming at designing robot mechanisms for these applications, we present in this paper a systematic enumeration of line-symmetric motion generators (LSMGs), i.e., robot mechanisms that generate the line-symmetric motion manifold $\mathrm{M}_{4}$, following a procedure based on symmetric space theory. LSMGs present a ubiquitous line symmetry of their joint axes, thus offering a new understanding of the line-symmetric motions.
\end{abstract}

Keywords: Line-symmetric motion, symmetric space, type synthesis, line-symmetric motion generator

\footnotetext{
A preliminary version of this paper was submitted for presentation at 16th International Symposium on Advances in Robot Kinematics, Bologna, Italy, July 1 - 5, 2018.

* Corresponding author

Email address: yuanqing.wu@unibo.it,marco.carriacto@unibo.it (Yuanqing $\mathrm{Wu}^{*}$ and Marco Carricato)
} 


\section{Nomenclature}

\begin{tabular}{|c|c|}
\hline$\ell, \ell_{0}, \ell(\mu), \ldots$ & lines represented by unit dual vectors \\
\hline LSMG & line-symmetric motion generator \\
\hline ICPM & interconnected parallel mechanism \\
\hline $\mathrm{SE}(3)$ & special Euclidean group \\
\hline $\mathfrak{s e}(3)$ & Lie algebra of $\mathrm{SE}(3)$ \\
\hline $\mathfrak{s e}(3)^{*}$ & dual space of $\mathfrak{s e}(3)$ (wrench space) \\
\hline $\mathbf{g}, \mathbf{h}, \ldots$ & elements of $\mathrm{SE}(3)$ \\
\hline $\boldsymbol{\xi}, \boldsymbol{\eta}, \ldots$ & twist vectors in $\mathfrak{s e}(3)$ \\
\hline$\zeta, \zeta_{i}, \ldots$ & wrench vectors in $\mathfrak{s e}(3)^{*}$ \\
\hline$\left[\boldsymbol{\xi}_{1}, \boldsymbol{\xi}_{2}\right]$ & commutator of $\boldsymbol{\xi}_{1}, \boldsymbol{\xi}_{2} \in \mathfrak{s e}(3)$ \\
\hline$s_{\mathbf{g}}: \mathrm{SE}(3) \rightarrow \mathrm{SE}(3)$ & inversion symmetry map associated with a point $\mathbf{g} \in \mathrm{SE}(3)$ \\
\hline $\mathbb{R} \mathrm{P}^{n}$ & $n$-dimensional real projective space \\
\hline$Q_{s}$ & Study quadric \\
\hline$\left[a_{0}: a_{1}: a_{2}: a_{3}: b_{0}: b_{1}: b_{2}: b_{3}\right]$ & Study parameters \\
\hline $\mathrm{M}_{4}$ & line-symmetric motion manifold \\
\hline $\mathfrak{m}_{4}$ & Lie-triple subsystem (LTS) of $\mathrm{M}_{4}$ \\
\hline $\mathfrak{h}_{4}$ & commutator algebra of $\mathfrak{m}_{4}$ \\
\hline $\mathrm{T}_{\mathrm{g}} \mathrm{M}_{4}$ & (right-trivialized) tangent space of $\mathrm{M}_{4}$ at $\mathrm{g}$ \\
\hline $1, \mathbf{i}, \mathbf{j}, \mathbf{k}$ & basis elements of the quaternion algebra \\
\hline$\varepsilon$ & dual number: $\varepsilon^{2}=0$ \\
\hline $\operatorname{Ad}(\mathbf{g})$ & Adjoint transformation by $\mathbf{g} \in \mathrm{SE}(3)$ \\
\hline$e^{\xi}$ & quaternion exponential of $\boldsymbol{\xi} \in \mathfrak{s e}(3)$ \\
\hline $\mathcal{L}$ & directed line space \\
\hline$\left(\boldsymbol{\xi}^{+}, \boldsymbol{\xi}^{-}\right)$ & symmetric twist pair (SP) of $\mathfrak{m}_{4}$ \\
\hline$\left(\boldsymbol{\xi}_{1}^{+}, \ldots, \boldsymbol{\xi}_{4}^{+}, \boldsymbol{\xi}_{4}^{-}, \ldots, \boldsymbol{\xi}_{1}^{-}\right)$ & symmetric twist chain (SC) of $\mathfrak{m}_{4}$ \\
\hline $\mathcal{M}^{-}, \mathcal{M}_{i}^{-}, \ldots$ & distal half of a $\mathfrak{m}_{4}-\mathrm{SC}$ \\
\hline $\mathcal{M}^{+}, \mathcal{M}_{i}^{+}, \ldots$ & proximal half of a $\mathfrak{m}_{4}-\mathrm{SC}$ \\
\hline$S^{-}, S_{i}^{-}, \ldots$ & twist subspace of $\mathcal{M}^{-}, \mathcal{M}_{i}^{-}, \ldots$ \\
\hline$(S)^{\perp}$ & reciprocal wrench subspace of a twist subspace $S$ \\
\hline
\end{tabular}

\section{Introduction}

When a rigid body is axially reflected through a moving line, its image undergoes a so-called line-symmetric motion. In other words, a line-symmetric motion is a one-parameter motion generated by half-turns of the reference body about a line $\ell$ undergoing a one-parameter motion [1], as illustrated in Fig. 1. A line-symmetric motion is uniquely determined by the basic surface [2, Ch. 9.7] swept out by $\ell(\mu), \mu \in \mathbb{R}^{+}$. Historically, line-symmetric motions were probably first studied by Krames [3] and are known as a rich source of special motions, such as the vertical Darboux motion, the Bricard motion, and the Bennett motion, and were extensively studied by using both basic surfaces and axodes $[2$, 1]. Line-symmetric motions found applications in characterizing self-motions of Griffis-Duffy type parallel manipulators [4] and also point-symmetric hexapods [5].

Other studies on line-symmetric motions focus on the structure and properties of the space of all line-symmetric motions that share a common initial 


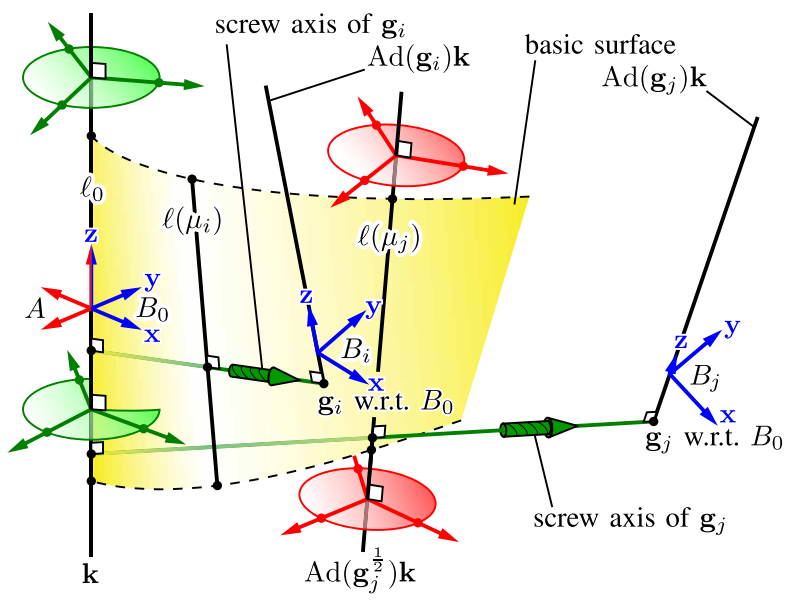

Figure 1: Line-symmetric motion about a moving line $\ell(\mu)$ parameterized by $\mu \in \mathbb{R}^{+}$. The half-turned image $B_{0}$ of frame $A$ about $\ell_{0}$ serves as the reference frame. Green and red arrows represent, respectively, screw axes of $\mathrm{T}_{1} \mathrm{M}_{4}=\mathfrak{m}_{4}$ and $\mathrm{T}_{\mathbf{g}_{j}} \mathrm{M}_{4}=\operatorname{Ad}\left(\mathbf{g}_{j}^{1 / 2}\right) \mathfrak{m}_{4}$.

line $\ell_{0}[1,6,7]$. In particular, we recently proved that this space, henceforth denoted $\mathrm{M}_{4}$, is a four-dimensional submanifold of the rigid displacement group (or special Euclidean group) SE(3), and is a symmetric subspace [6]. More precisely, as a symmetric space, $\mathrm{SE}(3)$ is a differentiable manifold that can be isometrically point-reflected onto itself over any point on the manifold [8]. The point-reflection map or inversion symmetry at a point $\mathbf{g} \in \mathrm{SE}(3)$, denoted $s_{\mathbf{g}}$, is defined as $s_{\mathbf{g}}(\mathbf{h})=\mathbf{g h}^{-1} \mathbf{g}, \forall \mathbf{h} \in \mathrm{SE}(3)$. A symmetric subspace is a submanifold that is closed under inversion symmetry. As we have shown in [6] and shall recall in the next section, the fact that $\mathrm{M}_{4}$ is a symmetric subspace gives rise to many important properties that will be useful for the particular application studied in this paper.

In this paper, we focus on the mechanism synthesis problem, namely, on the synthesis of 4-DoF mechanisms capable of generating arbitrary line-symmetric motions, or in other words, mechanisms whose motion manifolds are open submanifolds of $\mathrm{M}_{4}$. As far as we are aware, no such mechanisms exist in the literature. Therefore, this paper probably introduces for the first time a class of line-symmetric motion generators (LSMGs). As we have demonstrated in Fig. 1 and shall elaborate in Sect. 2.3, LSMGs can move a line-symmetric object from an initial to a goal configuration by screwing along the common perpendicular of the initial and the goal line, without incurring self spin or sliding about and along its own axis. This kind of motions has apparent applications in robotic motion planning and design for line-symmetric manipulation where only the shape of the line-symmetric object is of concern.

The paper is organized as follows. Sect. 2 gives a brief review of the motion manifold $\mathrm{M}_{4}$ and discusses its application in characterizing motion of objects with line symmetry. Sect. 3 proposes a special class of redundant kinematic 
chains, called symmetric chains, that may generate $\mathrm{M}_{4}$ under a symmetric movement condition. Sect. 4 implements the symmetric chains in the synthesis of a class of LSMGs with both in-parallel kinematic structure and interconnecting joints, which we refer to as interconnected parallel mechanisms. Finally, we conclude the paper with a discussion about possible followup work.

\section{Line-Symmetric Motion Manifold}

\subsection{Dual quaternion representation}

Following the notation in [1], an element $\mathbf{g}$ of the special Euclidean group $\mathrm{SE}(3)$ is mapped into the Study quadric $Q_{s}$ under dual quaternion representation:

$$
Q_{s}:=\left\{\mathbf{g}=\left(a_{0}+a_{1} \mathbf{i}+a_{2} \mathbf{j}+a_{3} \mathbf{k}\right)+\varepsilon\left(b_{0}+b_{1} \mathbf{i}+b_{2} \mathbf{j}+b_{3} \mathbf{k}\right) \in \mathbb{R} \mathrm{P}^{7} \mid \sum_{i=0}^{3} a_{i} b_{i}=0\right\}
$$

where $\mathbf{i}, \mathbf{j}, \mathbf{k}$ denote the quaternion units and $\varepsilon$ denotes the dual number $\left(\varepsilon^{2}=0\right)$. For elements $\mathbf{g}$ of $\mathrm{SE}(3), \sum_{i=0}^{3} a_{i}^{2} \neq 0$. We also identify elements of the Lie algebra $\mathfrak{s e}(3)$ of $\mathrm{SE}(3)$ with dual quaternion vectors:

$$
\mathfrak{s e}(3):=\left\{\left(a_{1} \mathbf{i}+a_{2} \mathbf{j}+a_{3} \mathbf{k}\right)+\varepsilon\left(b_{1} \mathbf{i}+b_{2} \mathbf{j}+b_{3} \mathbf{k}\right) \mid a_{i}, b_{i} \in \mathbb{R}, i=1,2,3\right\}
$$

We emphasize that the non-zero scalings of the homogeneous coordinates [ $a_{0}$ : $\left.a_{1}: a_{2}: a_{3}: b_{0}: b_{1}: b_{2}: b_{3}\right]$ all represent the same transformation $\mathbf{g}$. Thus it is convenient to assume that $\sum_{i=0}^{3} a_{i}^{2}=1$.

A half-turn $\ell$ is a rotation about a line for a magnitude of $\pi$. A line-symmetric motion parameterized by $\mu \in \mathbb{R}$ is given by:

$$
\mathbf{g}(\mu)=\ell(\mu) \ell_{0}^{-1}=\ell(\mu) \ell_{0}, \quad \ell_{0}=\ell(0)
$$

where it is apparent that $\ell_{0}^{-1}=\ell_{0}$ (Fig. 1). As illustrated in Fig. 1, $\mathbf{g}(\mu)$ is the screw motion along the common perpendicular of $\ell_{0}$ and $\ell(\mu)$ with magnitudes of rotation and translation specified, respectively, by twice the angle and distance between $\ell_{0}$ and $\ell(\mu)$. In other words, all finite screw axes of the line-symmetric motion manifold $\mathrm{M}_{4}$ perpendicularly intersect the characteristic line $\ell_{0}$, and their corresponding Plücker coordinates span the following 4D twist subspace:

$$
\mathfrak{m}_{4}=\left\{\boldsymbol{\xi}=\left(a_{1} \mathbf{i}+a_{2} \mathbf{j}\right)+\varepsilon\left(b_{1} \mathbf{i}+b_{2} \mathbf{j}\right) \mid a_{i}, b_{i} \in \mathbb{R}, i=1,2\right\}
$$

where $\ell_{0}$ is aligned with the $\mathbf{z}$-axis of the reference frame $B_{0}$. Moreover, $\mathrm{M}_{4}$ is the exponential image ${ }^{1}$ of $\mathfrak{m}_{4}$ :

$$
\mathrm{M}_{4}=\exp \mathfrak{m}_{4}:=\left\{e^{\boldsymbol{\xi}} \mid \forall \boldsymbol{\xi} \in \mathfrak{m}_{4}\right\}
$$

\footnotetext{
${ }^{1}$ For consistency, we assume in this paper that $e^{(\cdot)}$ is the quaternion exponential map; the screw motion $\mathbf{g}$ corresponding to a fixed unit screw axis $\boldsymbol{\xi}$ and magnitude $\theta$ is given by $e^{\frac{\theta}{2} \boldsymbol{\xi}}$. See [9] for more details.
} 


\subsection{Symmetric subspace property}

Further geometric properties of $\mathrm{M}_{4}$ may be derived by considering its symmetric subspace structure [6,7]. In particular, a symmetric subspace M of $\mathrm{SE}(3)$ is always given by the exponential image of a special type of vector subspace $\mathfrak{m}$ of $\mathfrak{s e}(3)$ called a Lie triple subsystem (LTS), namely one that satisfies:

$$
\left[\left[\boldsymbol{\xi}_{1}, \boldsymbol{\xi}_{2}\right], \boldsymbol{\xi}_{3}\right] \in \mathfrak{m} \quad \forall \boldsymbol{\xi}_{1}, \boldsymbol{\xi}_{2}, \boldsymbol{\xi}_{3} \in \mathfrak{m}
$$

where the commutator $[\cdot, \cdot]$ is defined by $\left[\boldsymbol{\xi}_{1}, \boldsymbol{\xi}_{2}\right]:=\boldsymbol{\xi}_{1} \times \boldsymbol{\xi}_{2}, \forall \boldsymbol{\xi}_{1}, \boldsymbol{\xi}_{2} \in \mathfrak{s e}(3)$.

The LTS $\mathfrak{m}_{4}$ of $\mathrm{M}_{4}$, as given in Eq. (4), comprises screws of arbitrary pitch along all lines that perpendicularly intersect the $\mathbf{z}$-axis (named the fifth special four-system in [10]). The commutator algebra of $\mathfrak{m}_{4}$, denoted $\mathfrak{h}_{4}$, is the cylindrical algebra along the z-axis:

$$
\mathfrak{h}_{4}:=\left[\mathfrak{m}_{4}, \mathfrak{m}_{4}\right]=\left\{a_{3} \mathbf{k}+\varepsilon b_{3} \mathbf{k} \mid a_{3}, b_{3} \in \mathbb{R}\right\}
$$

It is immediate that $\mathfrak{m}_{4} \cap \mathfrak{h}_{4}=\{0\}$ and $\mathfrak{m}_{4} \oplus \mathfrak{h}_{4}=\mathfrak{s e}(3)$. We summarize hereafter the symmetric subspace properties of $\mathrm{M}_{4}$, proved in [6], which will be useful in the next section:

1. Inversion symmetry: $\mathrm{M}_{4}$ is closed under inversion symmetry:

$$
\text { ghg } \in \mathrm{M}_{4}, \quad \forall \mathbf{g}, \mathbf{h} \in \mathrm{M}_{4}
$$

2. Conjugation invariance: $\mathrm{M}_{4}$ remains unchanged under arbitrary cylindrical displacement, i.e., invariant under conjugation by elements of the form $e^{\eta}, \boldsymbol{\eta} \in \mathfrak{h}_{4}$ :

$$
e^{\boldsymbol{\eta}} \mathrm{M}_{4} e^{-\boldsymbol{\eta}}=\mathrm{M}_{4} \quad \forall \boldsymbol{\eta} \in \mathfrak{h}_{4}
$$

or equivalently, $\mathfrak{m}_{4}$ is invariant under Adjoint transformations by elements of $\mathfrak{h}_{4}$ :

$$
\operatorname{Ad}\left(e^{\boldsymbol{\eta}}\right) \mathfrak{m}_{4}:=e^{\boldsymbol{\eta}_{\mathfrak{m}}} \mathfrak{m}_{4} e^{-\boldsymbol{\eta}}=\mathfrak{m}_{4} \quad \forall \boldsymbol{\eta} \in \mathfrak{h}_{4}
$$

3. Half-angle property: the (right-trivialized) tangent space of $\mathrm{M}_{4}$ at a generic point $\mathbf{g}=e^{\boldsymbol{\xi}} \in \mathrm{M}_{4}$, denoted $\mathrm{T}_{\mathbf{g}} \mathrm{M}_{4}$, is give by:

$$
\mathrm{T}_{\mathbf{g}} \mathrm{M}_{4}=\operatorname{Ad}\left(e^{\frac{\xi}{2}}\right) \mathfrak{m}_{4}
$$

In other words, $\mathrm{T}_{\mathbf{g}} \mathrm{M}_{4}$ is a displaced copy of $\mathfrak{m}_{4}$, with its characteristic line located at $\operatorname{Ad}\left(e^{\frac{\xi}{2}}\right) \mathbf{k}$, which exactly corresponds to the line on the basic surface generating the current transformation $\mathbf{g}$, as shown in Fig. 1.

4. Generalized polar decomposition: any transformation $\mathbf{g}$ in a local neighborhood of $1 \in \mathrm{SE}(3)$ may be uniquely written as the product of two exponentials with exponents in $\mathfrak{m}_{4}$ and $\mathfrak{h}_{4}$, respectively:

$$
\mathbf{g}=e^{\boldsymbol{\xi}} e^{\boldsymbol{\eta}} \quad \boldsymbol{\xi} \in \mathfrak{m}_{4}, \boldsymbol{\eta} \in \mathfrak{h}_{4}
$$




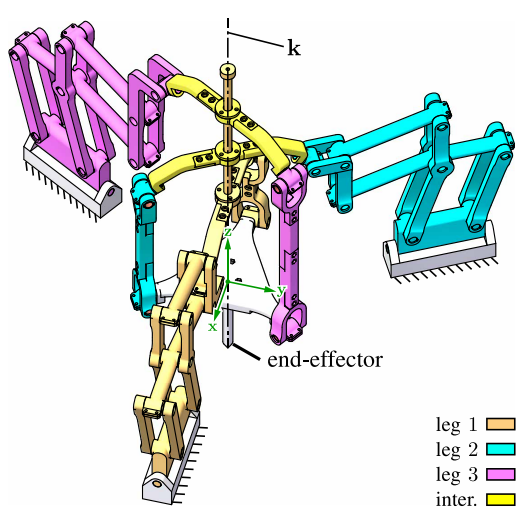

(a)

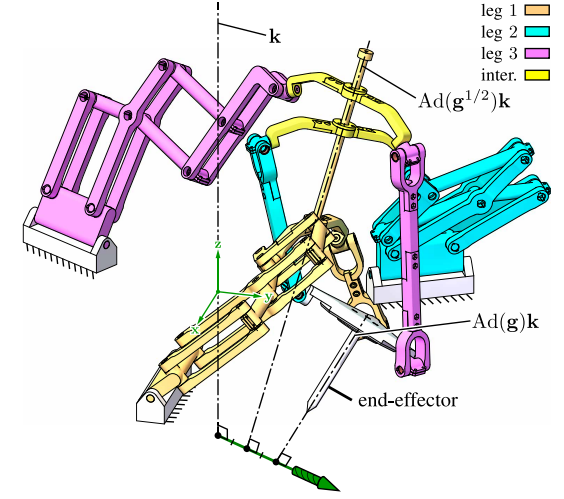

(b)

Figure 2: Demonstration of a LSMG (under development, courtesy of Roberto di Leva) for axis alignment of the stylus of a haptic interface. (a) Initial configuration; (b) target configuration.

\subsection{Motion properties of the LSMG from an application point of view}

\subsubsection{Application in haptic interface design}

It is clear that any screw axis belonging to the screw system of $\mathfrak{m}_{4}$ may serve as a finite screw axis for $\mathrm{M}_{4}$ and hence its LSMGs. Using the four DoFs of $\mathrm{M}_{4}$, we may displace a line-symmetric object with initial symmetry axis $\mathbf{k}$ so that its axis occupies an arbitrary location $\operatorname{Ad}\left(\mathbf{g}_{i}\right) \mathbf{k}, \mathbf{g}_{i} \in \mathrm{M}_{4}$ : it suffices to screw the object along the common perpendicular of $\mathbf{k}$ and $\operatorname{Ad}\left(\mathbf{g}_{i}\right) \mathbf{k}$ (see Fig. 1, where the finite screw axis is represented by a green threaded solid arrow). Alternatively, we see from the generalized polar decomposition Eq. (12) that for any $\mathbf{g} \in \mathrm{SE}(3)$ :

$$
\operatorname{Ad}(\mathbf{g}) \mathbf{k}=\operatorname{Ad}\left(e^{\boldsymbol{\xi}} e^{\boldsymbol{\eta}}\right) \mathbf{k}=\operatorname{Ad}\left(e^{\boldsymbol{\xi}}\right) \operatorname{Ad}\left(e^{\boldsymbol{\eta}}\right) \mathbf{k}=\operatorname{Ad}\left(e^{\boldsymbol{\xi}}\right) \mathbf{k}, \quad \boldsymbol{\xi} \in \mathfrak{m}_{4}, \boldsymbol{\eta} \in \mathfrak{h}_{4}
$$

where the cylindrical motion $e^{\boldsymbol{\eta}}, \boldsymbol{\eta} \in \mathfrak{h}_{4}$ does not contribute to the change of the overall shape (axis) of the object. This observation naturally leads to the following potential application of LSMGs. When tracking the stylus of a haptic interface [11], the six DoFs of the stylus can be functionally decomposed into $\mathrm{M}_{4}$ describing its axis location and the cylindrical motion group describing the stylus spin and penetration depth. A LSMG capable of arbitrarily positioning the end-effector (stylus) axis is shown in Fig. 2. The explanation of its structure is postponed to the next sections.

\subsubsection{Application in remote center-of-motion device design}

Recall that for any point on the characteristic line $\ell_{0}$ of $\mathrm{M}_{4}$, there passes a pencil of arbitrary-pitch finite screw axes (as illustrated by the green disks in Fig. 1), which in particular allows the end-effector of a LSMG to perform omnidirectional tilt about the point. In other words, a LSMG may work like a 2-DoF zero-torsion parallel wrist [12] about any point on $\ell_{0}$ (in its workspace). This property is graphically illustrated in Fig. 3 (a) and (b). The point of rotation is sometimes referred to as a remote center-of-motion (RCM). 


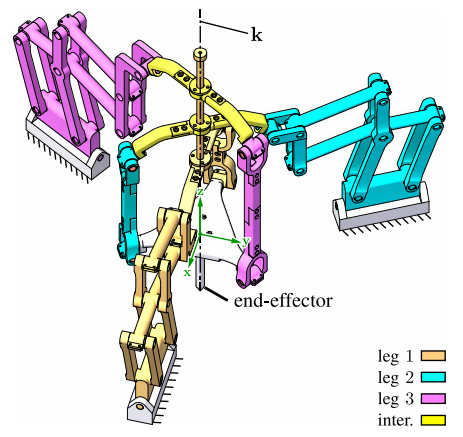

(a)

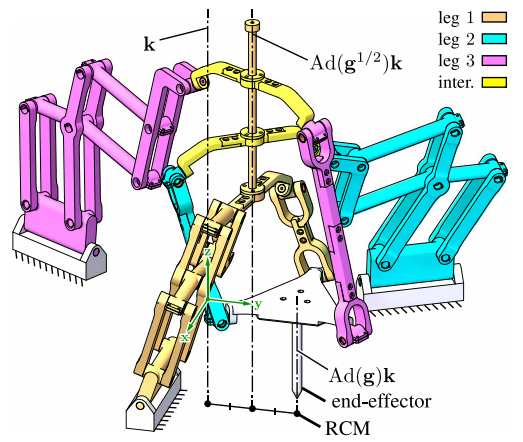

(c)

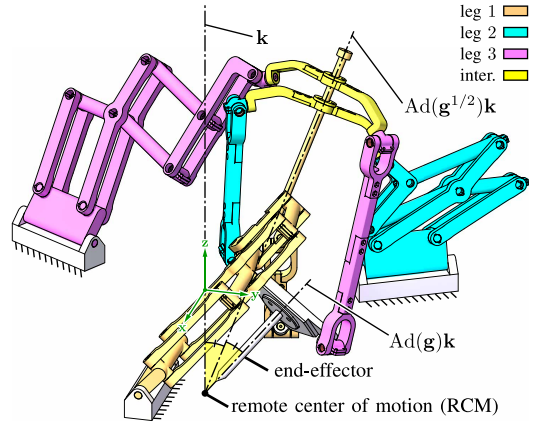

(b)

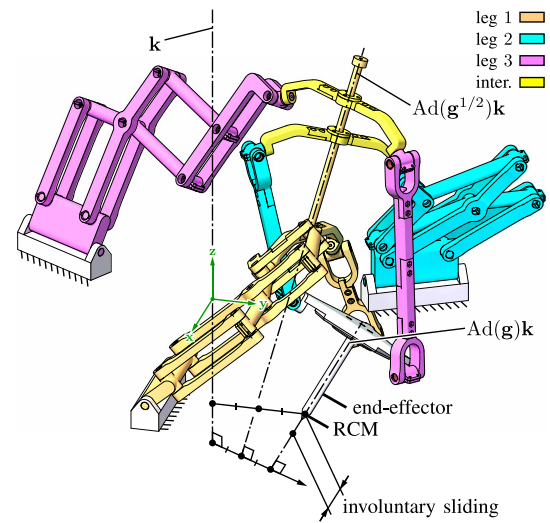

(d)

Figure 3: Demonstration of a LSMG as a RCM device for minimally invasive surgery. (a) Initial configuration; (b) tilting about a $\mathrm{RCM}$ on the characteristic line $\mathbf{k}$; (c) translating the end-effector over to a desired RCM lying outside the characteristic line $\mathbf{k}$; (d) tilting the end-effector about the desired RCM in (c) with involuntary sliding.

More generally, a LSMG can also place the RCM at an arbitrary location in space (within its workspace). As illustrated in Fig. 3 (c) and (d), the LSMG can first translate its end-effector above the intended RCM that does not lie on $\ell_{0}$ and then tilt its axis about the RCM. Note however that in this case there is an involuntary sliding of the end-effector along its axis. This can be easily compensated by an additional prismatic joint concatenated to the end-effector along its axis.

The aforesaid motion properties of LSMGs may be potentially applied to minimally invasive surgery (MIS), where a surgical instrument has to be inserted into patient's body through a small trocar. The instrument must be pivoted at the trocar without incurring lateral motions, i.e., considering the trocar as a $\mathrm{RCM}$ of the instrument [13]. One of the main problems of existing MIS parallel robot designs $[14,15]$ is that they can only move about a fixed RCM (however, see [16] for an exception). The whole robot must then be properly placed with 
respect to the patient so that the $\mathrm{RCM}$ is aligned with the intended trocar. On the other hand, the LSMG (with an additional cylindrical joint concatenated to its end-effector) may serve as a better alternative where the RCM can be easily adapted to the trocar on the patient without moving the whole mechanism.

\section{Type Synthesis of Kinematic Chains Generating Line Symmetric Motions}

\subsection{State-of-the-art parallel mechanism type synthesis methods}

State-of-the-art methods on type synthesis of mechanisms for motion generation are mainly based on the mechanical realization of product-of-exponential (POE) manifolds [6] (or a virtual chain [17]) by serial kinematic chains. Hunt was probably the first to give an account of type synthesis of parallel mechanisms by using screw theory [18]. Later Hervé et al. [19] proposed a systematic type synthesis method using Lie subgroups of SE(3), and later generalized it to include dependent products of subgroups [20, 21]. Screw-theory-based type synthesis methods were later pursued, among others, by Huang and Li [22], Kong and Gosselin [23, 24], Fang and Tsai [25] and Carricato [26, 27]. Synthesis approaches based on screw theory and Lie groups may eventually lead to the same results [28]. A thorough review of design methods for parallel mechanisms is out of the scope of this paper. The interested reader may refer to [17].

\subsection{Generating $\mathrm{M}_{4}$ using symmetric chains}

It can be proved that $\mathrm{M}_{4}$ is not a POE manifold and hence can be generated by no 4 -DoF serial kinematic chain. This impossibility is essentially due to the fact that the generalized polar decomposition Eq. (12) of a POE $e^{\boldsymbol{\xi}_{1}} e^{\boldsymbol{\xi}_{2}}$, $\boldsymbol{\xi}_{1}, \boldsymbol{\xi}_{2} \in \mathfrak{m}_{4}$, namely:

$$
e^{\boldsymbol{\xi}_{1}} e^{\boldsymbol{\xi}_{2}}=e^{\boldsymbol{\xi}^{\prime}} e^{\boldsymbol{\eta}} \quad \boldsymbol{\xi}^{\prime} \in \mathfrak{m}_{4}, \boldsymbol{\eta} \in \mathfrak{h}_{4}
$$

will in general have a non-zero $\boldsymbol{\eta}$-exponent when neither of $\boldsymbol{\xi}_{i}$ 's is a zero vector. It turns out that $\mathrm{M}_{4}$ can only be generated by redundant kinematic chains (i.e., having more than four 1-DoF lower pairs) with additional constraints imposed.

In [6], we introduced the concept of symmetric twist chains (SCs) for the generation of symmetric subspaces of $\mathrm{SE}(3)$. In particular, a $\mathfrak{m}_{4}$-SC is an 8-tuple

of unit twists, denoted as $\left(\boldsymbol{\xi}_{1}^{+}, \boldsymbol{\xi}_{2}^{+}, \boldsymbol{\xi}_{3}^{+}, \boldsymbol{\xi}_{4}^{+}, \boldsymbol{\xi}_{4}^{-}, \boldsymbol{\xi}_{3}^{-}, \boldsymbol{\xi}_{2}^{-}, \boldsymbol{\xi}_{1}^{-}\right)$, which comprises four nesting symmetric pairs $(\mathrm{SP})\left(\boldsymbol{\xi}_{i}^{+}, \boldsymbol{\xi}_{i}^{-}\right)$defined by:

$$
\left\{\begin{array}{l}
\boldsymbol{\xi}_{i}^{+}:=\boldsymbol{\xi}_{i}+\boldsymbol{\eta}_{i} \\
\boldsymbol{\xi}_{i}^{-}:=\boldsymbol{\xi}_{i}-\boldsymbol{\eta}_{i}
\end{array} \quad \boldsymbol{\xi}_{i} \in \mathfrak{m}_{4}, \boldsymbol{\eta}_{i} \in \mathfrak{h}_{4}, i=1, \ldots, 4\right.
$$

and satisfies one of the following equivalent conditions (see [6] for details):

$$
\begin{aligned}
& \operatorname{span}\left\{\boldsymbol{\xi}_{1}, \boldsymbol{\xi}_{2}, \boldsymbol{\xi}_{3}, \boldsymbol{\xi}_{4}\right\}=\mathfrak{m}_{4} \\
& \operatorname{span}\left\{\boldsymbol{\xi}_{1}^{+}, \boldsymbol{\xi}_{2}^{+}, \boldsymbol{\xi}_{3}^{+}, \boldsymbol{\xi}_{4}^{+}\right\} \oplus \mathfrak{h}_{4}=\mathfrak{s e}(3) \\
& \operatorname{span}\left\{\boldsymbol{\xi}_{4}^{-}, \boldsymbol{\xi}_{3}^{-}, \boldsymbol{\xi}_{2}^{-}, \boldsymbol{\xi}_{1}^{-}\right\} \oplus \mathfrak{h}_{4}=\mathfrak{s e}(3)
\end{aligned}
$$


Geometrically, each $\boldsymbol{\xi}_{i}^{+}$(after sign flip) is always line-symmetric to $\boldsymbol{\xi}_{i}^{-}$about the characteristic line $\ell_{0}$, as illustrated in Fig. 4. In other words, we have:

$$
\operatorname{Ad}(\mathbf{k}) \boldsymbol{\xi}_{i}^{+}=\mathbf{k}\left(\boldsymbol{\xi}_{i}+\boldsymbol{\eta}_{i}\right) \mathbf{k}^{-1}=\mathbf{k} \boldsymbol{\xi}_{i} \mathbf{k}^{-1}+\mathbf{k} \boldsymbol{\eta}_{i} \mathbf{k}^{-1}=-\boldsymbol{\xi}_{i}+\boldsymbol{\eta}_{i}=-\boldsymbol{\xi}_{i}^{-}
$$

where we have used the fact that $\boldsymbol{\xi}_{i} \in \mathfrak{m}_{4}$ perpendicularly intersects $\mathbf{k}$ and $\boldsymbol{\eta}_{i} \in \mathfrak{h}_{4}$ coincides with $\mathbf{k}$. Alternatively, every SP $\left(\boldsymbol{\xi}_{i}^{+}, \boldsymbol{\xi}_{i}^{-}\right)$can be built from $\boldsymbol{\xi}_{i} \in \mathfrak{m}_{4}$ via a geometric approach. For example, $\left(\boldsymbol{\xi}_{i}^{+}, \boldsymbol{\xi}_{i}^{-}\right)$is generated from $\boldsymbol{\xi}_{i} \in \mathfrak{m}_{4}$ (when $\boldsymbol{\xi}_{i}$ has a finite pitch $p_{i}$ ) by first applying a pair of rotations about $\boldsymbol{\delta}_{i} \in \mathfrak{m}_{4}$ and then a pair of translations along $\boldsymbol{\delta}_{i}^{\prime}$, both with equal and opposite magnitudes. When $\boldsymbol{\xi}_{j} \in \mathfrak{m}_{4}$ has instead infinite pitch, $\left(\boldsymbol{\xi}_{j}^{+}, \boldsymbol{\xi}_{j}^{-}\right)$is generated by applying a pair of rotations about $\boldsymbol{\delta}_{j} \in \mathfrak{m}_{4}$ with equal and opposite magnitudes. For future use, we denote the joint variable of $\boldsymbol{\xi}_{i}^{ \pm}$by $\theta_{i}^{ \pm}$.

The reason for the above definition of SCs is that such a kinematic chain may generate $\mathrm{M}_{4}$ under the following symmetric movement condition:

$$
\theta_{i}^{+}=\theta_{i}^{-}=: \theta_{i}, \quad i=1,2,3,4
$$

To prove this condition, we first notice that:

$$
\left(\theta_{1}, \theta_{2}, \theta_{3}, \theta_{4}\right) \mapsto e^{\frac{\theta_{1}}{2}} \boldsymbol{\xi}_{1}^{+} e^{\frac{\theta_{2}}{2} \boldsymbol{\xi}_{2}^{+}} e^{\frac{\theta_{3}}{2} \boldsymbol{\xi}_{3}^{+}} e^{\frac{\theta_{4}}{2} \boldsymbol{\xi}_{4}^{+}} e^{\frac{\theta_{4}}{2} \boldsymbol{\xi}_{4}^{-}} e^{\frac{\theta_{3}}{2} \boldsymbol{\xi}_{3}^{-}} e^{\frac{\theta_{2}}{2} \boldsymbol{\xi}_{2}^{-}} e^{\frac{\theta_{1}}{2} \boldsymbol{\xi}_{1}^{-}} \in \mathrm{M}_{4}
$$

as a result of the definition of SPs and the inversion symmetry (Eq. (8)) of $\mathrm{M}_{4}$. Second, the Jacobian of the above map at $(0,0,0,0)$ :

$$
\left(\dot{\theta}_{1}, \dot{\theta}_{2}, \dot{\theta}_{3}, \dot{\theta}_{4}\right) \mapsto \sum_{i=1}^{4} \frac{1}{2}\left(\boldsymbol{\xi}_{i}^{+}+\boldsymbol{\xi}_{i}^{-}\right) \dot{\theta}_{i}=\sum_{i=1}^{4} \boldsymbol{\xi}_{i} \dot{\theta}_{i}
$$

is a linear isomorphism since $\left\{\boldsymbol{\xi}_{i}\right\}_{i=1}^{4}$ are linearly independent by definition of $\mathrm{SC}$. Therefore, the $\mathfrak{m}_{4}$-SC generates $\mathrm{M}_{4}$ by Eq. (19) by inverse function theorem [29].

\subsection{Type synthesis of $\mathfrak{m}_{4}-S C s$}

We shall consider, for simplicity, synthesis of $\mathfrak{m}_{4}$-SCs with only revolute joints, which amounts to specifying a quadruple of 0-pitch screws $\mathcal{M}^{-}:=\left(\boldsymbol{\xi}_{4}^{-}\right.$, $\left.\boldsymbol{\xi}_{3}^{-}, \boldsymbol{\xi}_{2}^{-}, \boldsymbol{\xi}_{1}^{-}\right)$satisfying Eq. (16c):

$$
S^{-}:=\operatorname{span}\left\{\boldsymbol{\xi}_{4}^{-}, \boldsymbol{\xi}_{3}^{-}, \boldsymbol{\xi}_{2}^{-}, \boldsymbol{\xi}_{1}^{-}\right\}, \quad S^{-} \oplus \mathfrak{h}_{4}=\mathfrak{s e}(3)
$$

In general, the axes of all 0-pitch screws of a 4-dimensional twist space (a foursystem, in brief) form a two-parameter family of lines known as a linear congruence.

Following Hunt's classification of general and special four-systems [10], we have the following cases ${ }^{2}$ : 


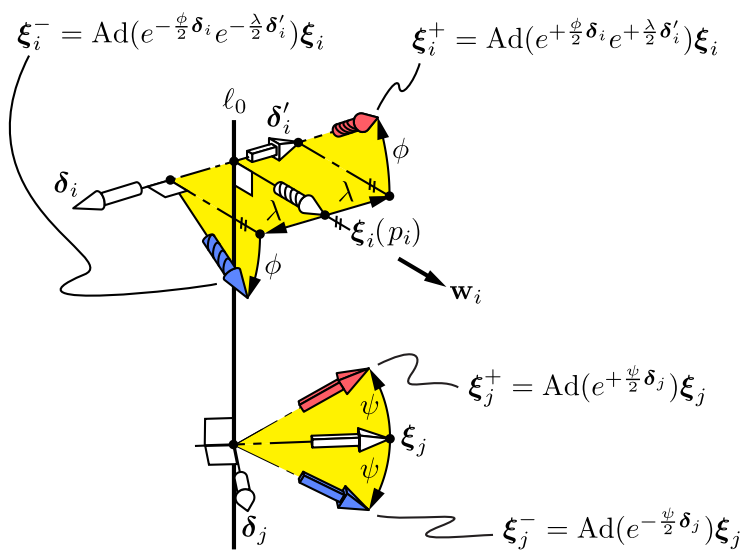

Figure 4: Examples of SPs of $\mathfrak{m}_{4}$. Round, prism and threaded arrows represent, respectively, 0 -pitch, infinite-pitch and finite-nonzero-pitch screws.

G) The collection of all 0-pitch screws of a general four-system comprises a linear congruence, which may:

$\mathrm{G}_{2}$ ) have all its lines meet two real skew lines called directrices (hyperbolic net) (Fig. 5(a)); the reciprocal wrench subspace $\left(S^{-}\right)^{\perp}$ is spanned by 0 -pitch screws $\boldsymbol{\zeta}_{1}$ and $\boldsymbol{\zeta}_{2}$ aligned with the directrices;

$\mathrm{G}_{1}$ ) have only one real directrix (parabolic net); the reciprocal wrench subspace $\left(S^{-}\right)^{\perp}$ is spanned by a finite nonzero-pitch screw $\zeta_{1}$ and a 0-pitch screw $\boldsymbol{\zeta}_{2}$ aligned with the directrix (Fig. 5(b));

$\mathrm{G}_{0}$ ) have two complex directrices (elliptic net); the congruence is a oneparameter family of reguli on $\infty^{1}$ coaxial hyperboloids (Fig. 6(a)).

I) The 0-pitch screws of a first special four-system may:

$\mathrm{I}_{0}$ ) comprise an axial-symmetric elliptic net if its reciprocal wrench system is formed by a pencil of finite-nonzero-pitch screws (Fig. 6(a));

$\mathrm{I}_{2}$ ) meet two intersecting real directrices, resulting in a degenerate congruence comprising a field of lines in the directrices' plane, as well as a bundle of lines with center at the intersection of the directrices (Fig. 6(b)).

II) The 0-pitch screws of a second special four-system form a degenerate congruence comprising:

\footnotetext{
${ }^{2}$ Case G refers to the general four-system, whereas cases I, II, III, IV and V refer to special four-systems of types 1 through 5 . A subscript 0,1 or 2 indicates the presence of, respectively, 0,1 or 2 real directrices.
} 


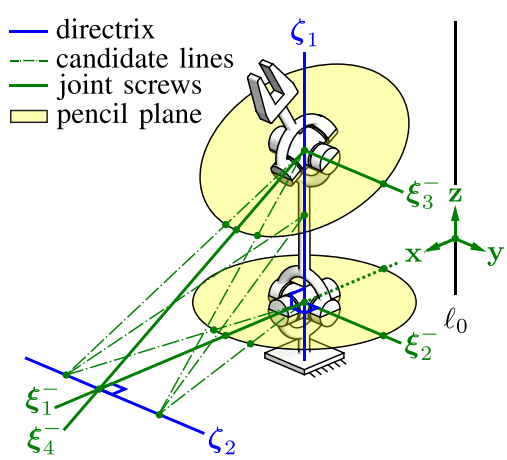

(a) type $\mathrm{G}_{2}$

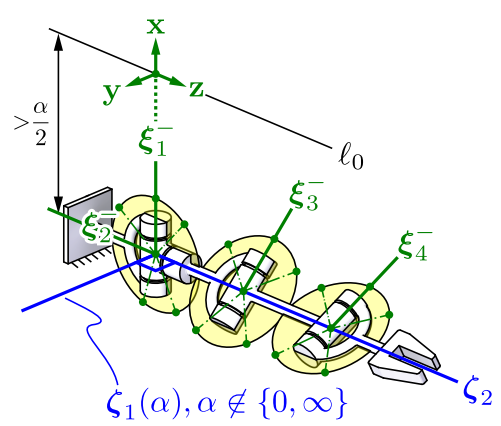

(b) type $\mathrm{G}_{1}$

Figure 5: Congruences formed by the axes of the 0-pitch screws in a general four-system, with their associated kinematic chains. In the absence of one or two real directrices, $\boldsymbol{\zeta}_{1}(\alpha)$ and $\zeta_{2}(\beta)$ are basis screws of the reciprocal two-system, with $\alpha$ and $\beta$ denoting the screw pitches. When $\alpha$ or $\beta$ is zero, the axis of the corresponding screw is a real directrix.

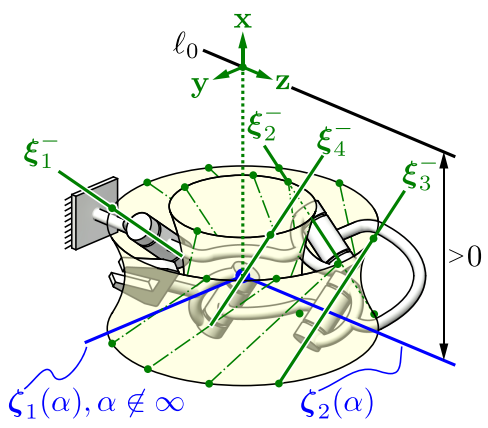

(a) type $\mathrm{I}_{0}\left(\mathrm{G}_{0}\right)$

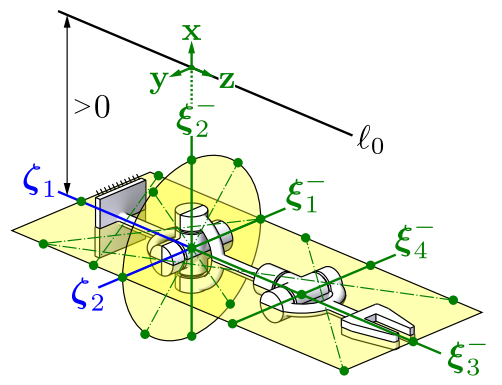

(b) type $\mathrm{I}_{2}$

Figure 6: Congruences formed by the axes of the 0-pitch screws in a first special four-system, with their associated kinematic chains.

$\mathrm{II}_{1}$ ) a one-parameter family of pencils of parallel lines on as many parallel planes, if its reciprocal wrench system is formed by a parallel pencil of finite-nonzero-pitch screws (Fig. 7(a)).

$\mathrm{II}_{2}$ ) a parallel bundle formed by all lines parallel to a real directrix and a field formed by all lines on a plane containing the real directrix (Fig. 7(b)).

III) The 0-pitch screws of a third special four-system (the Schönflies algebra) form a parallel bundle formed by all lines parallel to a given direction; there are no four linearly independent 0 -pitch screws.

IV) The 0-pitch screws of a fourth special four-system form a one-parameter family of pencils of concurrent lines on as many parallel planes, with their centers lying on a real directrix (Fig. 8(a)). 


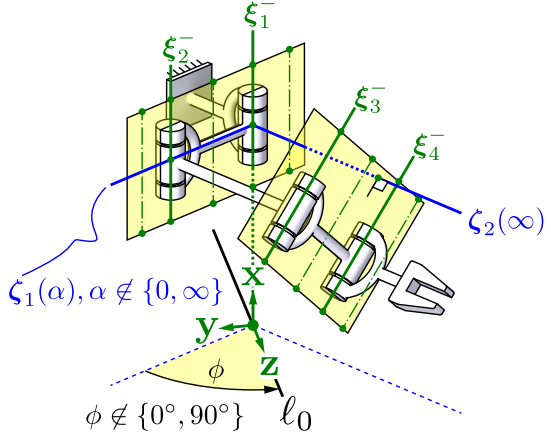

(a) type $\mathrm{II}_{1}$

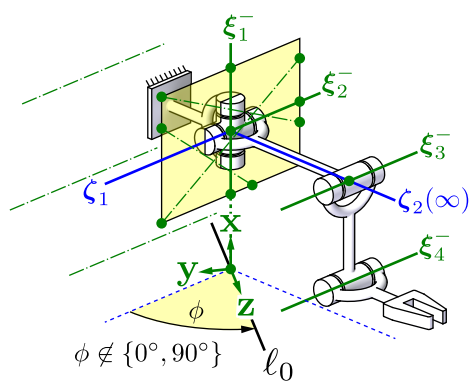

(b) type $\mathrm{II}_{2}$

Figure 7: Congruences formed by the axes of the 0-pitch screws in special four-systems of type 2 (a) and type 3 (b), with their associated kinematic chains.

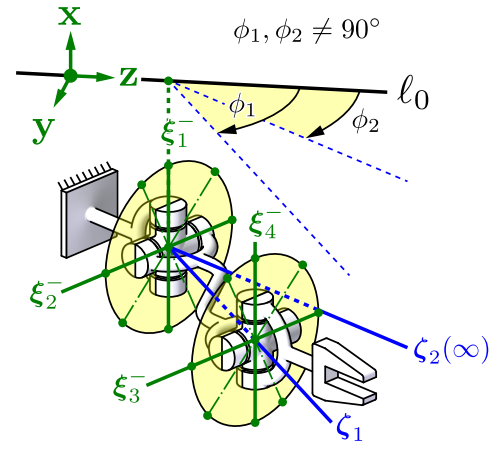

(a) type IV

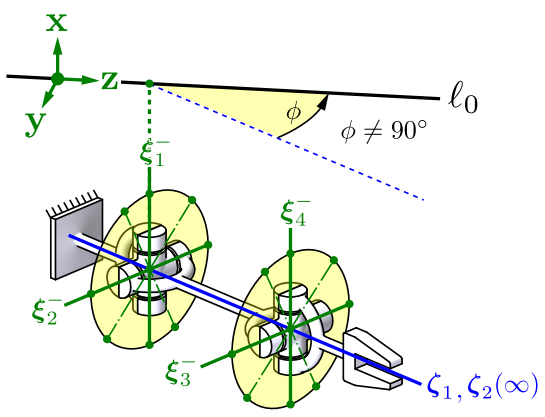

(b) type $\mathrm{V}$

Figure 8: Congruences formed by the axes of the 0-pitch screws in special four-systems of type IV (a) and type V (b), with their associated kinematic chains.

V) The 0-pitch screws of a fifth special four-system lie on all lines intersecting perpendicularly a real directrix (Fig. 8(b)).

The aforementioned cases, except for the third special four-system, lead to distinct $\mathfrak{m}_{4}$-SC types, which are summarized in Tab. 1 . We remark that the results we have obtained may be easily generalized to include prismatic joints, because it is straightforward to identify the infinite-pitch screws in the aforesaid foursystems.

\section{Type synthesis of line-symmetric motion generators}

\subsection{Synthesis procedure for ICPM-type LSMGs}

When the symmetric movement condition Eq. (18) is not imposed, $\mathfrak{m}_{4}$-SCs are essentially 8-DoF kinematic chains, and therefore cannot directly form a 
Table 1: Four-systems and their associated $\mathfrak{m}_{4}$-SCs comprising revolute $(\mathcal{R})$, universal $(\mathcal{U})$ and spherical $(\mathcal{S})$ joints.

\begin{tabular}{|c|c|c|c|}
\hline & congruence of 0 -pitch screws & $\mathcal{M}^{-}$ & condition (16c) \\
\hline $\mathrm{G}_{2}$ & $\begin{array}{l}2 \text { pencils with centers lying on the same } \\
\text { directrix }\end{array}$ & $\mathcal{U U}$ & \multirow{5}{*}{$\begin{array}{l}\text { the cylindroid } \\
\text { spanned by } \\
\zeta_{1} \text { and } \zeta_{2} \\
\text { contains no } \\
\text { axis that per- } \\
\text { pendicularly } \\
\text { intersects } \ell_{0}\end{array}$} \\
\hline $\mathrm{G}_{1}$ & $\begin{array}{l}3 \text { skew lines intersecting a } 4 \text { th one at } \\
\text { right-angle }\end{array}$ & $\mathcal{U R \mathcal { R }}$ & \\
\hline $\mathrm{G}_{0}$ & \multirow{2}{*}{$\begin{array}{l}3 \text { lines lying on a regulus of a } \\
\text { hyperboloid, and a } 4 \text { th one outside it }\end{array}$} & $4 \mathcal{R}$ & \\
\hline $\mathrm{I}_{0}$ & & $4 \mathcal{R}$ & \\
\hline $\mathrm{I}_{2}$ & $\begin{array}{l}\text { a bundle and a line not passing through } \\
\text { the bundle center (or equivalently two } \\
\text { non-coplanar pencils sharing a line) }\end{array}$ & $\begin{array}{l}\mathcal{R S} \\
(\mathcal{U U})\end{array}$ & \\
\hline $\mathrm{II}_{1}$ & $\begin{array}{l}2 \text { pairs of parallel lines lying in } 2 \text { parallel } \\
\text { planes }\end{array}$ & $4 \mathcal{R}$ & \multirow{3}{*}{$\begin{array}{l}\text { neither } \zeta_{1} \text { nor } \\
\zeta_{2} \text { is } \\
\text { perpendicular } \\
\text { to } \ell_{0}\end{array}$} \\
\hline $\mathrm{II}_{2}$ & $\begin{array}{l}\text { a pencil and } 2 \text { parallel lines parallel to the } \\
\text { pencil plane }\end{array}$ & $\mathcal{U R \mathcal { R }}$ & \\
\hline $\mathrm{IV}, \mathrm{V}$ & 2 pencils in 2 parallel planes & $\mathcal{U U}$ & \\
\hline
\end{tabular}

parallel mechanism with a $4 \mathrm{D}$ motion space such as $\mathrm{M}_{4}$, because internal motions will be introduced into the SCs that in general violate Eq. (18). This issue is ubiquitous for almost all symmetric subspace motion generators, and can be systematically resolved by adding internal interconnections within the parallel mechanism built from in-parallel connection of multiple SCs [30]. We shall refer to such mechanisms as interconnected parallel mechanisms (ICPMs).

Synthesis and analysis of spatial ICPM were seldom investigated in the literature [31], possibly due to a lack of understanding of its underlying principle, i.e., why the interconnections are needed and how to exploit them in type synthesis. In the following, we present a type synthesis procedure of ICPM-type LSMGs that hopefully shed some light on this issue. It is adapted from a synthesis procedure for general symmetric subspace motion generators presented in [30].

Given a parallel mechanism comprising $l \mathfrak{m}_{4}$-SCs, denoted $\mathcal{M}_{i}:=\left(\boldsymbol{\xi}_{i 1}^{+}, \ldots\right.$, $\left.\boldsymbol{\xi}_{i 4}^{+}, \boldsymbol{\xi}_{i 4}^{-}, \ldots, \boldsymbol{\xi}_{i 1}^{-}\right), i=1, \ldots, l$, the following procedure (see also Fig. 9) can be used to systematically synthesize ICPM-type LSMGs:

Step 1) distal half-SC synthesis Synthesize, for $i=1, \ldots, l$, a chain of four revolute joints represented by the quadruple $\mathcal{M}_{i}^{-}:=\left(\boldsymbol{\xi}_{i 4}^{-}, \boldsymbol{\xi}_{i 3}^{-}, \boldsymbol{\xi}_{i 2}^{-}\right.$, $\boldsymbol{\xi}_{i 1}^{-}$) satisfying Eq. (16c), namely

$$
S_{i}^{-} \oplus \mathfrak{h}_{4}=\mathfrak{s e}(3) \quad S_{i}^{-}:=\operatorname{span}\left\{\boldsymbol{\xi}_{i 4}^{-}, \boldsymbol{\xi}_{i 3}^{-}, \boldsymbol{\xi}_{i 2}^{-}, \boldsymbol{\xi}_{i 1}^{-}\right\}
$$

Step 2) whole SC synthesis Complete the $\mathfrak{m}_{4}$-SC using line symmetry, as shown in Fig. 4. This gives rise to a parallel mechanism comprising $l$ $\mathfrak{m}_{4}$-SCs which share the same characteristic line $\ell_{0}$. 
Step 3) constraint synthesis Verify the constraint synthesis condition at the home configuration:

$$
\sum_{i=1}^{l}\left(S_{i}^{-}\right)^{\perp}=\mathfrak{s e}(3)^{*}
$$

where $\left(S_{i}^{-}\right)^{\perp}$ denotes the reciprocal wrench subspace of $S_{i}^{-}$and $\mathfrak{s e}(3)^{*}$ is the $6 \mathrm{D}$ wrench space. This is the same as verifying that the parallel mechanism formed by the $l$ distal half-SCs $\left\{\mathcal{M}_{i}^{-}\right\}_{i=1}^{l}$ has mobility zero (in the home configuration).

Step 4) adding interconnection If Step 3) is satisfied, a LSMG is formed by connecting the innermost links (i.e., the link joining $\boldsymbol{\xi}_{i 4}^{-}$to $\boldsymbol{\xi}_{i 4}^{+}$) of the $\mathfrak{m}_{4}$-SCs $\mathcal{M}_{i}$ 's to each other, by way of cylindrical joints along $\ell_{0}$ (i.e., the $\mathbf{z}$-axis).

It can be seen from Step 3) and 4) that an ICPM-type LSMG is essentially formed by concatenation of two intertwined parallel mechanisms that are linesymmetric to each other. The constraint synthesis condition Eq. (22) ensures that the distal mechanism moves in unison with the proximal mechanism. We emphasize that although Eq. (22) is only verified in the home configuration, the motion properties of the resulting LSMG nevertheless hold for finite movements.

To carry out the synthesis of LSMGs, note that Step 1) was dealt with in Sect. 3, and Steps 2) and 4) are trivial. Therefore, we are only left with verifying Step 3). When $\left(S_{i}^{-}\right)^{\perp}$ may be spanned by two 0 - or $\infty$-pitch screws $\boldsymbol{\zeta}_{i 1}, \boldsymbol{\zeta}_{i 2}$, $i=1, \ldots, l$ (which is the case for type $\mathrm{G}_{2}, \mathrm{I}_{2}, \mathrm{II}_{2}, \mathrm{IV}$ and $\mathrm{V}$; the other cases are discussed later in the paper), Step 3) reduces to verifying Eq. (23) with a set of generators $\left(\boldsymbol{\zeta}_{11}, \boldsymbol{\zeta}_{12}, \ldots, \boldsymbol{\zeta}_{l 1}, \boldsymbol{\zeta}_{l 2}\right)$ :

$$
\operatorname{span}\left\{\boldsymbol{\zeta}_{11}, \boldsymbol{\zeta}_{12}, \ldots, \boldsymbol{\zeta}_{l 1}, \boldsymbol{\zeta}_{l 2}\right\}=\mathfrak{s e}(3)^{*}
$$

Thus, at least three $\mathfrak{m}_{4}$-SCs are required for the synthesis of LSMGs. In particular, when all $\left\{\boldsymbol{\zeta}_{i j}\right\}_{i, j=1,1}^{3,2}$ are 0 -pitch wrenches, Eq. (23) reduces to determining the linear independence of six spatial lines (possibly at infinity) [32, 33].

\subsection{On the linear independence of six lines}

For the sake of simplicity, consider a LSMG comprising three $\mathfrak{m}_{4}$-SCs of the same type and of identical geometry, i.e., each one being a displaced copy of a reference chain, say $\mathcal{M}_{0}=\left(\boldsymbol{\xi}_{01}^{+}, \boldsymbol{\xi}_{02}^{+}, \boldsymbol{\xi}_{03}^{+}, \boldsymbol{\xi}_{04}^{+}, \boldsymbol{\xi}_{04}^{-}, \boldsymbol{\xi}_{03}^{-}, \boldsymbol{\xi}_{02}^{-}, \boldsymbol{\xi}_{01}^{-}\right)$:

$$
\boldsymbol{\xi}_{i j}^{ \pm}=\operatorname{Ad}\left(\mathbf{g}_{i}\right) \boldsymbol{\xi}_{0 j}^{ \pm} \quad i=1,2,3, j=1,2,3,4
$$

It can be inferred from the fact that the three $\mathfrak{m}_{4}$-SCs must share the same characteristic line $\ell_{0}$ (the $\mathbf{z}$-axis) that the leg displacement $\mathbf{g}_{i}$ can only be a cylindrical motion about $\ell_{0}$. This is consistent with the line-symmetric motion manifold $\mathrm{M}_{4}$ being conjugation invariant under cylindrical motion (Property 2 in Sect. 2). 


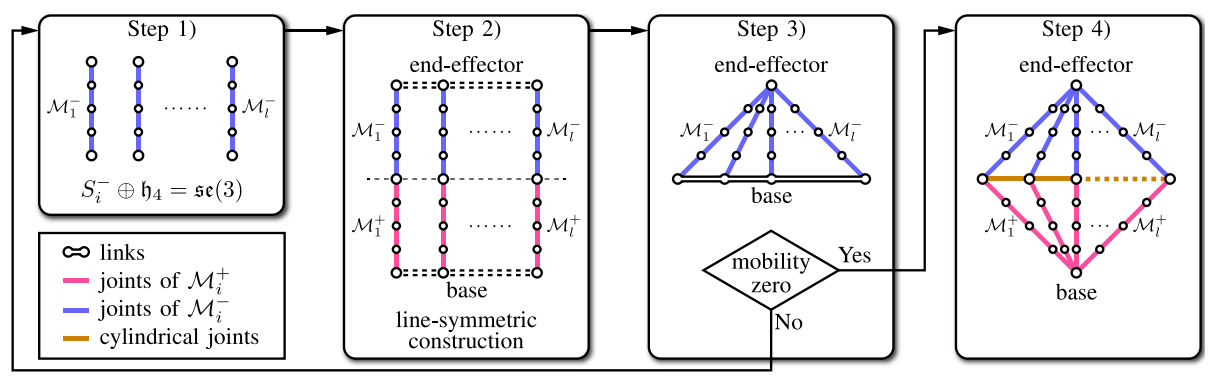

Figure 9: Synthesis procedure of line-symmetric motion generators.

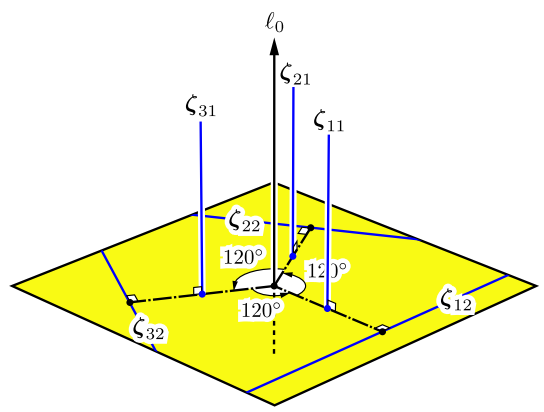

(a)

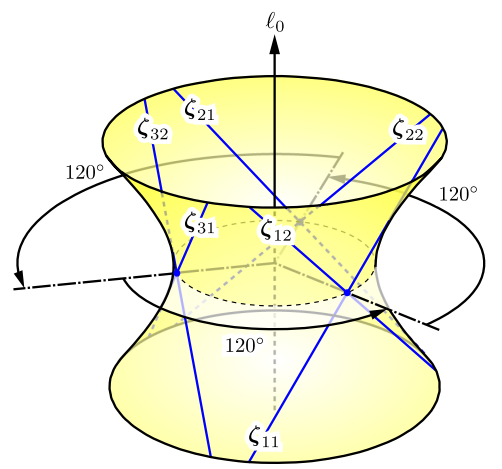

(b)

Figure 10: Spatial distribution of constraint wrenches of the LSMG. (a) A field and a parallel bundle perpendicular to it; (b) the two reguli on a ruled hyperboloid of revolution.

In principle, the three $\mathfrak{m}_{4}$-SCs of the LSMG can be generated by any cylindrical displacement. In order to have a uniform workspace distribution and singularity behavior, and also to simplify the geometry of the constraint wrenches $\left(\boldsymbol{\zeta}_{11}, \boldsymbol{\zeta}_{12}, \boldsymbol{\zeta}_{21}, \boldsymbol{\zeta}_{22}, \boldsymbol{\zeta}_{31}, \boldsymbol{\zeta}_{32}\right)$ for conceptual design, we displace the three $\mathfrak{m}_{4}$-SCs so that they form a three-fold line-symmetry about $\ell_{0}$. Consequently, both $\boldsymbol{\zeta}_{11}, \boldsymbol{\zeta}_{21}, \boldsymbol{\zeta}_{31}$ and $\boldsymbol{\zeta}_{12}, \boldsymbol{\zeta}_{22}, \boldsymbol{\zeta}_{32}$ form a three-fold line-symmetry about $\ell_{0}$.

\subsubsection{A field and a parallel bundle}

When $\boldsymbol{\zeta}_{i 1}, \boldsymbol{\zeta}_{i 2}$ form a pair of real finite lines, for $i=1,2,3$, the three $\mathfrak{m}_{4}$-SCs can be arranged so that, at the home configuration, $\boldsymbol{\zeta}_{11}, \boldsymbol{\zeta}_{21}$ and $\boldsymbol{\zeta}_{31}$ span a bundle of parallel lines (parallel to the z-axis), whereas $\boldsymbol{\zeta}_{12}, \boldsymbol{\zeta}_{22}$ and $\boldsymbol{\zeta}_{32}$ span a field in a plane (xy-plane) perpendicular to the parallel bundle (see Fig. 10(a)).

To see that these six lines are linearly independent, it suffices to see that the screw systems of the field and the parallel bundle comprise only 0- and $\infty$-pitch screws. Should the corresponding wrench subspaces, namely $\operatorname{span}\left(\boldsymbol{\zeta}_{11}, \boldsymbol{\zeta}_{21}, \boldsymbol{\zeta}_{31}\right)$ and $\operatorname{span}\left(\boldsymbol{\zeta}_{12}, \boldsymbol{\zeta}_{22}, \boldsymbol{\zeta}_{32}\right)$ have a non-trivial intersection, it must also comprise 0or $\infty$-pitch screws only. However, this is impossible because: the 0 -pitch screws of the field lie inside its plane while those of the bundle are perpendicular to it; 


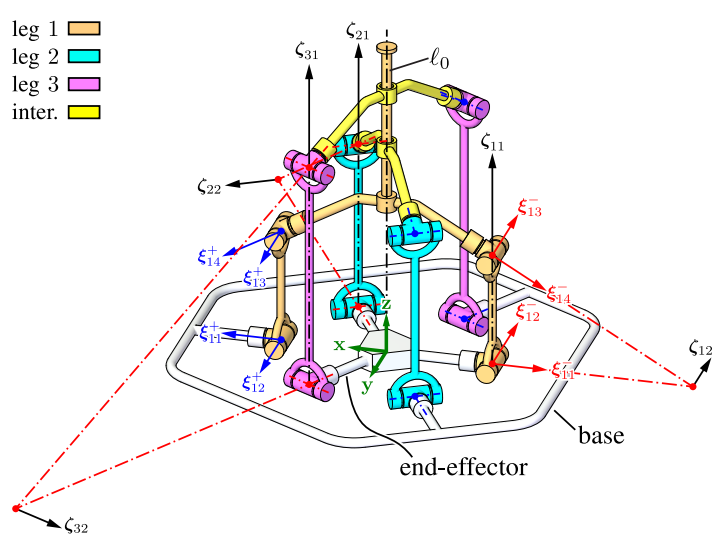

(a)

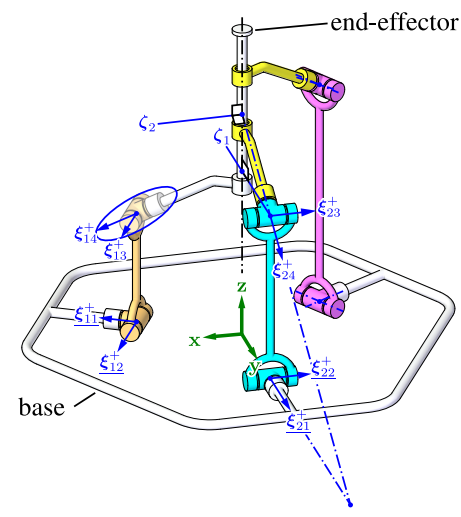

(b)

Figure 11: (a) Line symmetric motion generator of type $\mathrm{G}_{2}$. For clarity, only the joint screws of the first leg are shown; (b) actuation of the proximal half mechanism of the LSMG (actuated joint indicated by underline on the joint twist).

the $\infty$-pitch screws of the field are perpendicular to its plane while those of the bundle are parallel to it. Physically, the parallel bundle constrains the instantaneous end-effector motion onto the plane perpendicular to the bundle. On the other hand, the planar field further removes all instantaneous mobility inside

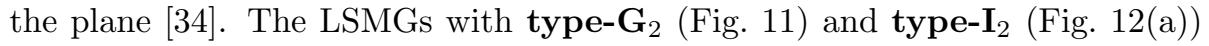
SCs may be synthesized in such a fashion. We would like to point out that the prototype design shown earlier in Fig. 2 and 3 is a type- $\mathrm{G}_{2}$ LSMG, with $\boldsymbol{\xi}_{i 2}^{+}, i=1,2,3$ replaced by double-parallelogram curve-scribing mechanism [35] (to avoid link collision).

\subsubsection{Two reguli on a ruled hyperboloid of revolution}

Another convenient spatial pattern for six linearly independent lines can be established using the two reguli on a ruled hyperboloid. It is well known that any three distinct lines belonging to either regulus is linearly independent. We may then simply set $\boldsymbol{\zeta}_{11}, \boldsymbol{\zeta}_{21}, \boldsymbol{\zeta}_{31}$ and $\boldsymbol{\zeta}_{12}, \boldsymbol{\zeta}_{22}, \boldsymbol{\zeta}_{32}$ to be distinct lines belonging to the two reguli of a ruled quadric of revolution respectively, as shown in Fig. 10(b). To see that all six lines are linearly independent, we notice that they give rise to a quadratic complex of tangent lines of the quadric [36, Ch. 16.91]. However, the six lines are linearly dependent only if they belong to a common linear complex. Note that in this case, the two constraint wrenches associated with the same $\mathfrak{m}_{4}$-SC form a pencil (such as in the case of type $\mathbf{I}_{2} \mathrm{SCs}$ ). This offers new types of LSMG other than the one shown in Fig. 12(a).

\subsubsection{Two reguli on concentric hyperboloids of revolution}

We may further generalize the previous case by suitably changing the common pitch of $\boldsymbol{\zeta}_{11}, \boldsymbol{\zeta}_{21}, \boldsymbol{\zeta}_{31}$ and/or $\boldsymbol{\zeta}_{12}, \boldsymbol{\zeta}_{22}, \boldsymbol{\zeta}_{32}$, or even let the two groups lie on different concentric hyperboloids, thus including all $\mathfrak{m}_{4}$-SCs with non-zero-pitch 


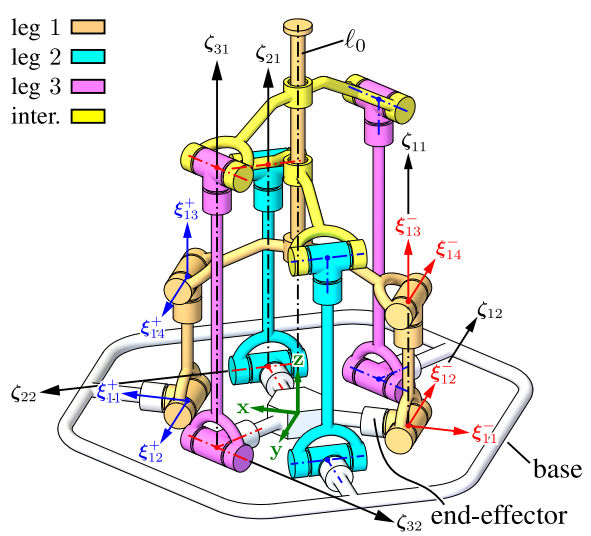

(a)

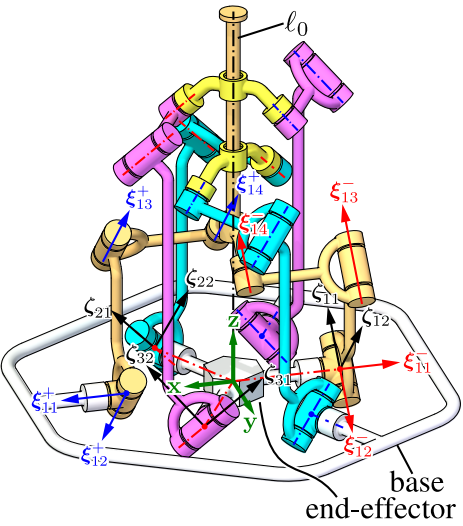

(b)

Figure 12: (a) Line symmetric motion generator of type $\mathrm{I}_{2}$; line symmetric motion generator of type $\mathrm{II}_{2}$. For clarity, only the joint screws of the first leg are shown.

constraint wrenches (type $\mathbf{G}_{0}, \mathbf{G}_{1}, \mathbf{I}_{0}$ and $\mathbf{I I}_{1}$ ). This is because the principle screws of the three-systems corresponding to $\boldsymbol{\zeta}_{11}, \boldsymbol{\zeta}_{21}, \boldsymbol{\zeta}_{31}$ and $\boldsymbol{\zeta}_{12}, \boldsymbol{\zeta}_{22}, \boldsymbol{\zeta}_{32}$ are perfectly aligned, which makes identification of singular configurations trivial. For example, changing the pitches of $\boldsymbol{\zeta}_{11}, \boldsymbol{\zeta}_{21}, \boldsymbol{\zeta}_{31}$ to a non-zero finite value $\alpha$ amounts changing the three principle pitches of $\operatorname{span}\left(\boldsymbol{\zeta}_{11}, \boldsymbol{\zeta}_{21}, \boldsymbol{\zeta}_{31}\right)$ by $\alpha$.

In the extreme case, $\boldsymbol{\zeta}_{i 2}(i=1,2,3)$ can be chosen to have an infinite pitch, so that $\boldsymbol{\zeta}_{12}, \boldsymbol{\zeta}_{22}, \boldsymbol{\zeta}_{32}$ span a bundle of infinite-pitch screws, and $\boldsymbol{\zeta}_{11}, \boldsymbol{\zeta}_{21}, \boldsymbol{\zeta}_{31}$ (after a convenient translation to become concurrent) span a bundle of 0-pitch screws. The LSMGs with type $\mathbf{I I}_{2}$ (Fig. 12(b)),type IV (Fig. 13(a)) and type V (Fig. 13(b)) SCs may be synthesized in such a fashion.

\subsection{Actuation of LSMGs}

It is clear from our synthesis approach that each LSMG comprises two intertwined parallel mechanisms. In order to fully control a LSMG, we may simply actuate four $\mathcal{R}$ joints of the proximal half mechanism so that, when the actuated joints are removed, the mechanism becomes a structure (thus achieving mobility zero) [17].

Consider for example the $\mathrm{G}_{2}$-type LSMG shown in Fig. 11 (a). Its proximal half mechanism, illustrated in Fig. 11 (b), comprises one 4-DoF leg $\mathcal{M}_{1}^{+}$and two 6-DoF legs, namely $\mathcal{M}_{2}^{+}$and $\mathcal{M}_{3}^{+}$each one being concatenated with a cylindrical joint along the z-axis at the home configuration. Theoretically speaking, the four DoFs of the two cylindrical joints in legs 2 and 3 are guaranteed to fully actuate the proximal half mechanism, since the mechanism reduces to the inparallel connection of $\mathcal{M}_{1}^{+}, \mathcal{M}_{2}^{+}$and $\mathcal{M}_{3}^{+}$after the two cylindrical joints are removed. Since, by construction, the in-parallel connection of $\mathcal{M}_{1}^{-}, \mathcal{M}_{2}^{-}$and $\mathcal{M}_{3}^{-}$has zero mobility, its line-symmetric image, namely that of $\mathcal{M}_{1}^{+}, \mathcal{M}_{2}^{+}$and $\mathcal{M}_{3}^{+}$should also have zero mobility. Note that this actuation strategy applies to other LSMGs synthesized in the paper. 


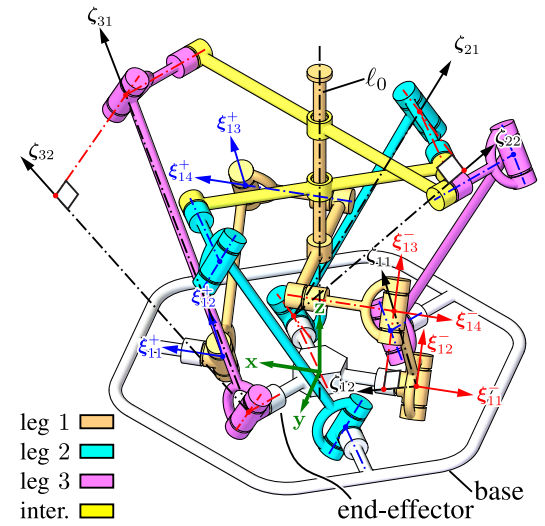

(a)

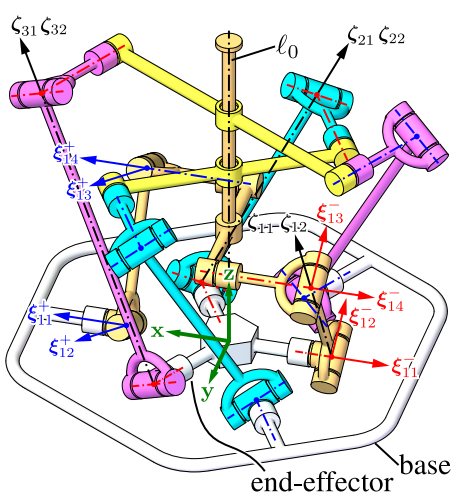

(b)

Figure 13: (a) Line symmetric motion generator of type IV; (b) line symmetric motion generator of type V. For clarity, only the joint screws of the first leg are shown.

However, from a practical point of view, it is preferable to actuate the joints located on the base of the LSMG. Therefore, a second actuation strategy may be proposed. Take again the $\mathrm{G}_{2}$-type LSMG for example, we may choose to actuate the first two revolute joints of the first two legs, namely $\boldsymbol{\xi}_{11}^{+}, \boldsymbol{\xi}_{12}^{+}, \boldsymbol{\xi}_{21}^{+}$and $\boldsymbol{\xi}_{22}^{+}$ (indicated by an underscore in Fig. 11 (b)), of the proximal half mechanism. To see that the removal of these four joints reduces the mechanism to a structure, we must verify that the screw systems of the two legs have a trivial intersection at the home configuration (since the third leg has $6 \mathrm{DoFs}$ ). This can be achieved by first identifying the two directrices, also denoted $\boldsymbol{\zeta}_{1}$ and $\boldsymbol{\zeta}_{2}$ in Fig. 11 (b), of the screw system of leg 2 , and then verifying that no zero-pitch screws of the pencil spanned by $\boldsymbol{\xi}_{13}^{+}$and $\boldsymbol{\xi}_{14}^{+}$(indicated by the disk with blue boundary in Fig. 11) would simultaneously intersect $\boldsymbol{\zeta}_{1}$ and $\boldsymbol{\zeta}_{2}$. In general, there is only one zero-pitch screw intersecting both directrices and also lying on the plane of the pencil, which cannot at the same pass the center of the pencil in a generic way. This means that a generic parametric design of the mechanism would lead to the proposed actuation strategy being valid. Each actuation pair $\left(\boldsymbol{\xi}_{11}^{+}, \boldsymbol{\xi}_{12}^{+}\right)$and $\left(\boldsymbol{\xi}_{21}^{+}, \boldsymbol{\xi}_{22}^{+}\right)$can be driven, for example, by a fully decoupled 2-DoF parallel wrist [37]. Finally, it can be shown that this actuation strategy also applies to other LSMGs synthesized in the paper.

\subsection{On completeness of the synthesis results}

So far, we have presented, based on a complete classification of four-systems (Tab. 1), axis-symmetric LSMGs with identical types of legs. Under such an assumption, the constraint synthesis problem of the LSMG reduces to one of the three geometry settings discussed in Sect. 4.2.1, 4.2.2 and 4.2.3. Although only the LSMGs with five of the nine types of $\mathfrak{m}_{4}$-SCs listed in Tab. 1 are illustrated in Fig. 11, 12 and 13, the remaining LSMGs may be proved to follow similar geometry. Generalizing from the synthesis results we have presented, 
one may then combine three arbitrary types of $\mathfrak{m}_{4}$-SCs chosen from Tab. 1, and also remove the axis-symmetry assumption to generate more synthesis results in a straightforward manner, albeit with laborious effort. When a LSMG is not constructed with axis-symmetry, the geometry of its constraint synthesis problem also presents a general pattern, which can still be properly conducted by algebraic computation instead of the geometric reasoning offered in Sect. 4.2.1, 4.2.2 and 4.2.3.

Another possible generalization is the replacement of one or more $\mathcal{R}$ joints in the synthesized LSMGs with prismatic joints. This should be done by following the same synthesis procedure presented in Sect. 3. The directions of the prismatic joints in the distal half $\mathfrak{m}_{4}$-SCs $\mathcal{M}_{i}^{-}, i=1, \ldots, l$ can be easily determined by the two constraint wrenches of the corresponding leg screw system. For example, for the type- $\mathrm{G}_{2}$ half $\mathrm{SC}$ shown in Fig. 5(a), there can be only one linearly independent prismatic joint which is perpendicular to both $\boldsymbol{\zeta}_{1}$ and $\boldsymbol{\zeta}_{2}$. When there is an infinite-pitch constraint wrench (as in the case of the type-- $\mathrm{I}_{1}$ half SC as illustrated in Fig. 7(a)), a total of two linearly independent prismatic joints can be found, which are both perpendicular to the constraint wrench with finite pitch. We remark that, due to the line-symmetric construction of the $\mathfrak{m}_{4}$-SCs, the prismatic joints appear in each $\mathrm{SC}$ in pairs. From a practical point of view, only one of the prismatic joints (in the proximal half SC) will be actuated, leaving unactuated prismatic joints in the resulting LSMG. Consequently, implementation of prismatic joints in a LSMG is arguably a less practical strategy.

\section{Conclusions}

We presented for the first time an essentially complete type synthesis of 4-DoF mechanisms capable of generating arbitrary line-symmetric motions, based on both symmetric space theory and classic screw theory. The synthesis conditions for the LSMGs are given, and are used to systematically generate a whole spectrum of novel mechanisms. As far as we consider synthesis using only revolute joints and three-fold line-symmetry, the synthesis result of the LSMGs presented here is complete. Our future work shall focus on conceptual design, analysis, optimization and prototyping of some of the mechanisms presented here.

\section{References}

[1] J. Selig, M. Husty, Half-turns and line symmetric motions, Mechanism and Machine Theory 46 (2) (2011) 156-167.

[2] O. Bottema, B. Roth, Theoretical kinematics, North-Holland Publishing Company, Amsterdam-New York-Oxford, 1979.

[3] J. Krames, Über fußpunktkurven von regelflächen und eine besondere klasse von raumbewegungen, Monatshefte für Mathematik und Physik 45 (1) (1936) 394-406. 
[4] A. Karger, M. Husty, Classification of all self-motions of the original Stewart-Gough platform, Computer-Aided Design 30 (3) (1998) 205-215.

[5] G. Nawratil, On the self-mobility of point-symmetric hexapods, Symmetry 6 (4) (2014) 954-974.

[6] Y. Wu, H. Löwe, M. Carricato, Z. Li, Inversion symmetry of the Euclidean group: theory and application to robot kinematics, IEEE Transactions on Robotics 32 (2) (2016) 312-326.

[7] Y. Wu, M. Carricato, Identification and geometric characterization of Lie triple screw systems and their exponential images, Mechanism and Machine Theory 107 (2017) 305-323.

[8] O. Loos, Symmetric spaces, Benjamin, 1969.

[9] J. M. Selig, Geometric fundamentals of robotics, Springer, 2004.

[10] K. H. Hunt, Kinematic geometry of mechanisms, Oxford University Press, 1978.

[11] L. J. Stocco, S. E. Salcudean, F. Sassani, Optimal kinematic design of a haptic pen, IEEE/ASME Transactions on Mechatronics 6 (3) (2001) 210220.

[12] Y. Wu, M. Carricato, Synthesis and singularity analysis of N-UUU parallel wrists: A symmetric space approach, Journal of Mechanisms and Robotics 9 (5) (2017) 051013.

[13] C.-H. Kuo, J. S. Dai, Robotics for minimally invasive surgery: a historical review from the perspective of kinematics, in: International Symposium on History of Machines and Mechanisms, Springer, 2009, pp. 337-354.

[14] C.-H. Kuo, J. S. Dai, Kinematics of a fully-decoupled remote center-ofmotion parallel manipulator for minimally invasive surgery, Journal of Medical Devices 6 (2) (2012) 021008.

[15] Q. Li, J. M. Hervé, P. Huang, Type synthesis of a special family of remote center-of-motion parallel manipulators with fixed linear actuators for minimally invasive surgery, Journal of Mechanisms and Robotics 9 (3) (2017) 031012 .

[16] D. Pisla, B. Gherman, C. Vaida, M. Suciu, N. Plitea, An active hybrid parallel robot for minimally invasive surgery, Robotics and ComputerIntegrated Manufacturing 29 (4) (2013) 203-221.

[17] X. Kong, C. M. Gosselin, Type synthesis of parallel mechanisms, Springer, 2007.

[18] K. Hunt, Constant-velocity shaft couplings: a general theory, Journal of Engineering for Industry 95 (2) (1973) 455-464. 
[19] J. Hervé, F. Sparacino, Structural synthesis of parallel robots generating spatial translation, in: Proceedings of the 5th IEEE international conference on advanced robotics, 1991, pp. 808-813.

[20] J. M. Hervé, The planar-spherical kinematic bond: implementation in parallel mechanisms, http://www.parallemic.org/Reviews/review013. html (2003).

[21] C.-C. Lee, J. M. Hervé, Translational parallel manipulators with doubly planar limbs, Mechanism and Machine Theory 41 (4) (2006) 433-455.

[22] Z. Huang, Q. Li, Type synthesis of symmetrical lower-mobility parallel mechanisms using the constraint-synthesis method, The International Journal of Robotics Research 22 (1) (2003) 59-79.

[23] X. Kong, C. M. Gosselin, Type synthesis of 3-dof translational parallel manipulators based on screw theory, Journal of Mechanical Design 126 (1) (2004) 83-92.

[24] X. Kong, C. M. Gosselin, Type synthesis of 3-dof spherical parallel manipulators based on screw theory, Journal of Mechanical Design 126 (1) (2004) 101-108.

[25] Y. Fang, L.-W. Tsai, Structure synthesis of a class of 4-dof and 5-dof parallel manipulators with identical limb structures, The International Journal of Robotics Research 21 (9) (2002) 799-810.

[26] M. Carricato, Decoupled and homokinetic transmission of rotational motion via constant-velocity joints in closed-chain orientational manipulators, Journal of Mechanisms and Robotics 1 (4) (2009) 041008.

[27] M. Carricato, D. Zlatanov, Persistent screw systems, Mechanism and Machine Theory 73 (2014) 296-313.

[28] J. Meng, G. Liu, Z. Li, A geometric theory for analysis and synthesis of sub6 dof parallel manipulators, IEEE Transactions on Robotics 23 (4) (2007) 625-649.

[29] W. M. Boothby, An introduction to differentiable manifolds and Riemannian geometry, Vol. 120, Academic press, 1986.

[30] Y. Wu, M. Carricato, Symmetric subspace motion generators, IEEE Transactions on Robotics, DOI: 10.1109/TRO.2018.2813377.

[31] M. Zoppi, D. Zlatanov, R. Molfino, On the velocity analysis of interconnected chains mechanisms, Mechanism and Machine Theory 41 (11) (2006) $1346-1358$.

[32] J.-P. Merlet, Singular configurations of parallel manipulators and Grassmann geometry, The International Journal of Robotics Research 8 (5) (1989) 45-56. 
[33] H. Pottmann, M. Peternell, B. Ravani, An introduction to line geometry with applications, Computer-Aided Design 31 (1) (1999) 3-16.

[34] D. L. Blanding, Exact Constraint: Machine Design Using Kinematic Processing, ASME press, 1999.

[35] G. J. Hamlin, A. C. Sanderson, Tetrobot: A modular approach to reconfigurable parallel robotics, Vol. 423, Springer, 2013.

[36] D. M. Y. Sommerville, Analytical geometry of three dimensions, Cambridge University Press, 2016.

[37] M. Carricato, V. Parenti-Castelli, A novel fully decoupled two-degreesof-freedom parallel wrist, The International Journal of Robotics Research 23 (6) (2004) 661-667. 